Published in final edited form as:

Int J Nurs Stud. 2014 October ; 51(10): 1366-1372. doi:10.1016/j.ijnurstu.2014.02.004.

\title{
Identification of Symptom Clusters among Patients with Heart Failure: An International Observational Study
}

\author{
Debra K. Moser, DNSc, RN, FAAN, \\ Professor and Gill Endowed Chair, University of Kentucky, College of Nursing \\ Kyoung Suk Lee, PhD, RN, \\ Assistant Professor, University of Wisconsin, School of Nursing \\ Jia-Rong Wu, PhD, RN, \\ Assistant Professor, University of North Carolina-Chapel Hill, School of Nursing \\ Gia Mudd-Martin, PhD, RN, \\ Assistant Professor, University of Kentucky, College of Nursing \\ Tiny Jaarsma, PhD, RN, FAAN, \\ Professor, Institute of Social and Welfare Studies, Faculty of Health Sciences, University of \\ Linköping
}

Tsuey-Yuan Huang, PhD, RN,

Associate Professor, Chang Gung University of Science and Technology, College of Nursing

Xui-Zhen Fan, PhD, RN,

Professor, Shandong University, School of Nursing

Anna Strömberg, PhD, RN,

Professor and Head of Division of Nursing, Department of Medicine and Health Sciences, Linköping University and Research Coordinator, Department of Cardiology, Linköping University Hospital

Terry A. Lennie, PhD, RN, FAAN, and

Professor and Associate Dean for PhD Program, University of Kentucky, College of Nursing

Barbara Riegel, DNSc, RN, FAAN

Professor and Edith Clemmer Steinbright Chair of Gerontology University of Pennsylvania, School of Nursing

\section{Abstract}

\author{
(C) 2014 Elsevier Ltd. All rights reserved. \\ Corresponding Author: Debra K. Moser, DNSc, RN, FAAN, Professor and Gill Endowed Chair, 527 CON, 751 Rose Street, \\ Lexington, KY 40536-0232, 859-323-6687, FAX 859-323-1057, dmoser@uky.edu. \\ Publisher's Disclaimer: This is a PDF file of an unedited manuscript that has been accepted for publication. As a service to our \\ customers we are providing this early version of the manuscript. The manuscript will undergo copyediting, typesetting, and review of \\ the resulting proof before it is published in its final citable form. Please note that during the production process errors may be \\ discovered which could affect the content, and all legal disclaimers that apply to the journal pertain.
}


Background-Virtually all patients with heart failure experience multiple symptoms simultaneously, yet clinicians and researchers usually consider symptoms in isolation. Recognizing and responding early to escalating symptoms is essential to preventing hospitalizations in heart failure, yet patients have considerable difficulty recognizing symptoms. Identification of symptom clusters could improve symptom recognition, but cultural differences may be present that must be considered.

Objectives-To identify and compare symptom clusters in heart failure patients from the United States, Europe and Asia.

Design-Cross-sectional, observational study.

Settings-In- and out-patient settings in three regions of the world: Asia (i.e., China and Taiwan); Europe (i.e., the Netherlands and Sweden); and the United States.

Participants-A total of 720 patients with confirmed heart failure. Propensity scoring using New York Heart Association Classification was used to match participants from each of the three regions.

Methods-Symptoms were identified using the Minnesota Living with Heart Failure Questionnaire. To identify symptom clusters we used cluster analysis with the hierarchical cluster agglomerative approach. We used the Euclidean distance to measure the similarity of variables. Proximity between groups of variables was measured using Ward's method. The resulting clusters were displayed with dendrograms, which show the proximity of variables to each other on the basis of semi-partial R-squared scores.

Results-There was a core group of symptoms that formed two comparable clusters across the countries. Dyspnea, difficulty in walking or climbing, fatigue/increased need to rest, and fatigue/low energy were grouped into a cluster, which was labeled as a physical capacity symptom cluster. Worrying, feeling depressed, and cognitive problems were grouped into a cluster, which was labeled as an emotional/cognitive symptom cluster. The symptoms of edema and trouble sleeping were variable among the countries and fell into different clusters.

Conclusion-Despite the diversity in cultures studied, we found that symptoms clustered similarly among the cultural groups. Identification of similar symptoms clusters among patients with heart failure may improve symptom recognition in both patients and healthcare providers.

\section{Keywords}

heart failure; symptom clusters; cognitive symptoms; emotional symptoms; physical symptoms

Heart failure (HF) is a growing health concern throughout the world. In the United States alone, the estimated prevalence of HF is around 5.8 million (Roger et al., 2012). Heart failure incidence has not declined for two decades (Roger et al., 2012). Heart failure affects at least 15 million of the 900 million persons in the 51 European countries represented by the European Society of Cardiology (Stromberg and Dickstein, 2008). Approximately 40\% of these patients are dead or readmitted within one year of hospital discharge (Stromberg and Dickstein, 2008). Despite limited knowledge about HF epidemiology in Asian countries, it is clear that HF prevalence is increasing along with the aging of that population and 
increased survival of patients experiencing coronary events (Alla et al., 2007, Jiang and Ge, 2009).

Information about the patient symptom experience is essential because efficacious symptom management is a major focus in HF. An important feature of the symptom experience in HF is that patients experience multiple symptoms simultaneously, and these symptoms are associated with detrimental outcomes (Bekelman et al., 2007, Blinderman et al., 2008, Ekman et al., 2005, Heo et al., 2008, Jurgens et al., 2009, Lee et al., 2010, Song et al., 2010, Zambroski et al., 2005). Thus, investigating co-occurring symptoms as symptom clusters may assist health care providers to better understand the symptom experience of patients with HF thereby allowing them to perform more effective clinical management.

A symptom cluster consists of two or more co-occurring symptoms (Miaskowski et al., 2007). Although symptoms within a cluster are strongly related, they do not necessarily share a common etiology (Miaskowski et al., 2007). There is increasing evidence to suggest that symptom clusters may be more predictive of outcomes than singly occurring symptoms.

Symptoms are conceptualized as subjective phenomenon reflecting perceived alterations in normal function (Dodd et al., 2001, Rhodes and Watson, 1987). Functional or structural abnormalities in body organs and systems are reflected as symptoms. The reality of the person in the context of his or her cultural and personal situation is a major component of symptom expression (Good and Good, 1981). Thus, patients with HF may have different symptom experiences and symptom clusters depending on their cultural background. For this reason, it is meaningful to perform international comparisons of symptom clusters and identify any differences. Accordingly, our specific aim was to identify and compare symptom clusters in the United States, Europe, and Asia.

\section{Methods}

This study was an observational, comparative investigation of differences in symptom clusters in each of regions studies. We included data from the United States, Europe (the Netherlands and Sweden) and Asia (China and Taiwan).

\section{Patients and settings}

Purposive sampling was used to recruit patients with HF from in- and out-patient settings in five countries: China, the Netherlands, Sweden, Taiwan, and the United States from 2008 through 2011. They were eligible for the study if they met the following selection criteria: 1) confirmed diagnosis of HF with either preserved or non-preserved systolic function; 2) no admission for myocardial infarction or stroke within the previous 3 months because these conditions substantially alter the typical HF symptom experience; 3) free of serious debilitating comorbidities such as end-stage renal or liver disease; 4) free of cognitive impairments that might hinder ability to participate in an interview with research nurses; and 5) on a cardiac transplantation waiting list. 


\section{Procedures}

This study was approved by the Institutional Review Boards or their equivalent at all participating sites. Investigators or trained research nurses who were native to each country recruited patients. Patients were referred for recruitment by nurses and physicians caring for them. Informed, signed consent was received from patients. Patients completed questionnaire packets. Sociodemographic and clinical data were obtained by patient interview and medical record review.

\section{Measurement}

Heart failure symptoms-Symptoms were identified from the Minnesota Living with Heart Failure Questionnaire (MLHFQ),(Rector et al., 1987) which assesses health-related quality of life in patients with HF. The instrument contains six physical symptom and three psychological symptom items that influence health-related quality of life: edema, dyspnea, difficulty walking or climbing stairs, fatigue/increased need to rest, fatigue/low energy, sleep difficulties, worrying, feeling depressed, and cognitive problems (difficulty concentrating or remembering things). Patients were asked to rate the impact of each item on a scale from 0 (no impact) to 5 (very severe impact). These items reflected the presence or absence of given symptoms, and the degree of negative impact caused by the symptoms, thus reflecting multiple dimensions of symptoms.

The instrument was translated from English to Chinese, Swedish, or Dutch by nativespeaking researchers, back-translated into English by a second native-speaking researcher, and examined for equality of meaning. In this study, Cronbach's alpha was used to assess reliability of the eight items and was found to be high at 0.88 for Asia, 0.90 for the United States, and 0.84 for Europe.

Sociodemographic and clinical characteristics-Patients were interviewed and their medical record examined to obtain the following sociodemographic and clinical data: age, gender, marital status, comorbidities, New York Heart Association (NYHA) functional classification, and medication.

\section{Statistical analyses}

Data were analyzed using SAS (version 9.2). Propensity scores were calculated using logistic regression. New York Heart Association functional class was used as a predictor to estimate propensity scores because NYHA functional class is associated with the symptom experience (Ekman et al., 2005). Because there were three regional groups (i.e., Asia, Europe, America), each patient from Asia was matched to one patient from Europe, and then each European patient who was selected from the previous matching technique was matched to one patient from the United States. The matching was done with the absolute difference between propensity scores of $+/-0.05$. By using this technique, comparable patient groups, 240 patients from each regional group, were identified for cluster analysis.

To identify symptom clusters we used cluster analysis, which maximizes the homogeneity of variables within clusters while simultaneously maximizing the heterogeneity between clusters (Everitt, 1972). The hierarchical cluster agglomerative approach was used. This 
technique treats each variable as a separate cluster at the beginning and then combines the variables into consecutively larger clusters based on their similarity. We used the Euclidean distance to measure the similarity of variables. Proximity between groups of variables was measured using Ward's method by which clusters were joined by minimizing the total within-cluster error sum of squares.

The resulting clusters were displayed with dendrograms, which show the proximity of variables to each other on the basis of semi-partial R-squared scores. Smaller values of semipartial R-squared scores indicate a higher degree of homogeneity of variables between clusters. The optimal number of clusters was determined based on dendrograms, the pseudo$F$ statistic, and the pseudo- $T$ squared statistic (Everitt et al., 2001).

\section{Results \\ Sample characteristics}

Demographic and clinical characteristics are presented in Table 2. Patients in Europe were older and less likely to have hypertension compared to patients in the United States and Asia. A larger percentage of patients in Asia were married than patients in the other regions.

\section{Symptom clusters}

There was a core group of symptoms that formed two comparable clusters across the countries. The symptoms of edema and trouble sleeping were variable among the countries and fell into different clusters.

United States-Three distinct cluster solutions emerged based on the dendrogram (Figure 1). The pseudo- $F$ statistic and the pseudo- $T$ squared statistic were used to examine whether the number of clusters based on the dendrogram was appropriate or not. These two statistics agreed that three solutions were optimal. Dyspnea, difficulty in walking or climbing, fatigue/ increased need to rest, and fatigue/low energy were grouped into a cluster, which was labeled as a physical capacity symptom cluster. Worrying, feeling depressed, cognitive problems, and sleep difficulties were grouped into a cluster, which was labeled as an emotional/cognitive symptom cluster. Edema did not cluster with either of these and formed a third unique solution, which was not deemed a cluster as it consisted of only one symptom.

Asia-Three distinct cluster solutions were identified based on the dendrogram (Figure 2). The pseudo- $F$ statistic and the pseudo- $T$ squared statistic also suggested three cluster solutions were optimal. The first cluster was identical to the physical capacity symptom cluster seen in the United States sample with one exception. It consisted of dyspnea, difficulty in walking or climbing, fatigue/increased need to rest, and fatigue/low energy as in the United States, but also included sleep difficulties. The second cluster formed an emotional/cognitive cluster and consisted of worrying, feeling depressed, and cognitive problems. Edema, as in the United States, formed a third solution that again, was not deemed a cluster as it consisted of only one symptom.

Europe-The dendrogram suggested three distinct cluster solutions (Figure 3). Three cluster solutions were suggested based on the pseudo- $F$ statistic and the pseudo-T squared 
statistic. As in the United States and Asia, there was a physical capacity symptom cluster, consisting of dyspnea, difficulty in walking or climbing, fatigue/increased need to rest, and fatigue/low energy. An emotional/cognitive symptom cluster consisted of worrying, feeling depressed, and cognitive problems. Finally, edema and sleep difficulties clustered together to form a third cluster.

\section{Discussion}

Despite the diversity in cultures reflected in the sample for this study, we found that symptoms clustered similarly among the cultural groups studied. There were two major symptoms clusters identified that were common to all countries. These findings suggest that the pathophysiologic genesis of HF symptom clusters is the same across cultures and their expression is not affected by culture.

Many investigators have examined the relationship of individual symptoms to outcomes (Ekman et al., 2005, Friedman, 1997, Friedmann et al., 2006, Smith et al., 2012). Individual symptoms, particularly dyspnea and depressive symptoms, are related to rehospitalization and mortality risk (Adams et al., 2012, Ekman et al., 2005, Johnson et al., 2012). These data are important for focusing attention on the importance of examining the symptom experience in heart failure. Our findings extend these data to demonstrate that a focus on individual symptoms may not be sufficient to assist patients or healthcare providers adequately assess for and recognize symptoms of escalating heart failure.

Data from many different patient populations demonstrate that symptoms commonly occur together, that clustering of symptoms together puts patients at higher risk for poor outcomes including higher morbidity and mortality, and that such risk is not clearly evident when symptoms are considered in isolation (Doering et al., 2009, Ferreira et al., 2008, Teunissen et al., 2006). For example, clustering of anxiety and depression in patients with coronary artery disease is associated with higher mortality that is the presence of anxiety or depression alone (Doering et al., 2009). The presence of higher numbers of symptoms confers greater mortality risk in patients with cancer. In one study, patients with four symptoms were nine times more likely to die as those with only one symptom (Teunissen et al., 2006).

Our findings suggest a universality of symptom expression and symptom clustering that is helpful to clinicians as they attempt to determine the meaning of symptoms in their patients with HF, and as they attempt to educate their patients to respond appropriately to symptoms (Jurgens et al., 2009). Knowledge of symptom clusters may also assist clinicians in risk stratification of their patients. We previously identified the presence of the same symptom clusters in another U.S. sample, and demonstrated that presence of the emotional/cognitive symptom cluster in patients was associated with increased risk of rehospitalization and mortality (Lee et al., 2010). Thus, our data suggest that regardless of cultural background, patients who present with an emotional/cognitive clustering of symptoms should be treated with extra vigilance given their increased risk of poor outcomes. The increased risk associated with the emotional/cognitive symptom cluster exists even in the absence of physical symptoms (Lee et al., 2010). 
Of interest, edema did not cluster with the larger physical symptom cluster in any country. In the United States and Asia, it did not cluster with any other symptom, and in Europe it clustered only with sleep difficulties. Edema may be unique among symptoms of HF because it is not noted for causing great distress unless extreme. For this reason it likely does not cluster with the other equally common, but more distressing symptoms of HF.

Limitations of this study include possible lack of generalizability to ethnic or cultural groups other than American and European Caucasians, and Asians. Another potential limitation is the use of an instrument, the MLHFQ that measures presence of, and distress related to, symptoms, but not other dimensions (i.e., frequency or severity) of the symptom experience. We used this instrument given the lack of reliable and valid instruments, at the time of the study, which included both physical and psychological symptoms common in patients with HF. There is evidence, however, that distress may best capture the negative impact of the symptom experience and provide a good source for determining how symptoms cluster (Cooley et al., 2003, Tishelman et al., 2005).

\section{Conclusion}

We found that the symptom experience for patients with heart failure across widely varying cultures is very similar. Among people from Asian, European and American backgrounds, symptoms occur in clusters and not in isolation. Given the difficulty patients have recognizing their own symptoms of escalating heart failure, the identification of symptom clusters may improve their ability to assess for and recognize symptoms so that they can act in a more timely manner to avert rehospitalization (Ryan et al., 2007, Smith et al., 2009)

\section{Acknowledgments}

Study Funding: This study was funded in part by a Center grant to the University of Kentucky, College of Nursing from NIH, NINR, 1P20NR010679 (Debra Moser, principal investigator); NIH, NINR, R01NR009280 (Terry Lennie, principal investigator); and NIH, NINR, R01 NR008567 (Debra Moser, principal investigator).

\section{References}

Adams J, Kuchibhatla M, Christopher EJ, Alexander JD, Clary GL, Cuffe MS, Califf RM, Krishnan RR, O'Connor CM, Jiang W. Association of depression and survival in patients with chronic heart failure over 12 Years. Psychosomatics. 2012; 53 (4):339-346. [PubMed: 22281436]

Alla F, Zannad F, Filippatos G. Epidemiology of acute heart failure syndromes. Heart Fail Rev. 2007; 12 (2):91-95. [PubMed: 17450426]

Bekelman DB, Havranek EP, Becker DM, Kutner JS, Peterson PN, Wittstein IS, Gottlieb SH, Yamashita TE, Fairclough DL, Dy SM. Symptoms, depression, and quality of life in patients with heart failure. J Card Fail. 2007; 13 (8):643-648. [PubMed: 17923356]

Blinderman CD, Homel P, Billings JA, Portenoy RK, Tennstedt SL. Symptom distress and quality of life in patients with advanced congestive heart failure. J Pain Symptom Manage. 2008; 35 (6):594603. [PubMed: 18215495]

Cooley ME, Short TH, Moriarty HJ. Symptom prevalence, distress, and change over time in adults receiving treatment for lung cancer. Psychooncology. 2003; 12 (7):694-708. [PubMed: 14502594]

Dodd M, Janson S, Facione N, Faucett J, Froelicher ES, Humphreys J, Lee K, Miaskowski C, Puntillo K, Rankin S, Taylor D. Advancing the science of symptom management. J Adv Nurs. 2001; 33 (5): 668-676. [PubMed: 11298204] 
Doering LV, Moser DK, Riegel B, McKinley S, Davidson P, Baker H, Meischke H, Dracup K. Persistent comorbid symptoms of depression and anxiety predict mortality in heart disease. Int $\mathbf{J}$ Cardiol. 2009

Ekman I, Cleland JG, Swedberg K, Charlesworth A, Metra M, Poole-Wilson PA. Symptoms in patients with heart failure are prognostic predictors: insights from COMET. J Card Fail. 2005; 11 (4):288-292. [PubMed: 15880338]

Ekman I, Cleland JG, Swedberg K, Charlesworth A, Metra M, Poole-Wilson PA. Symptoms in patients with heart failure are prognostic predictors: insights from COMET. Journal of cardiac failure. 2005; 11 (4):288-292. [PubMed: 15880338]

Everitt BS. Cluster analysis: a brief discussion of some of the problems. Br J Psychiatry. 1972; 120 (555):143-145. [PubMed: 5043719]

Everitt, BS.; Landau, S.; Leese, M. Cluster analysis. Arnold, a member of the Hodder Headline Group; New York: 2001.

Ferreira KA, Kimura M, Teixeira MJ, Mendoza TR, da Nobrega JC, Graziani SR, Takagaki TY. Impact of cancer-related symptom synergisms on health-related quality of life and performance status. J Pain Symptom Manage. 2008; 35 (6):604-616. [PubMed: 18362059]

Friedman MM. Older adults' symptoms and their duration before hospitalization for heart failure. Heart and Lung. 1997; 26 (3):169-176. [PubMed: 9176684]

Friedmann E, Thomas SA, Liu F, Morton PG, Chapa D, Gottlieb SS. Relationship of depression, anxiety, and social isolation to chronic heart failure outpatient mortality. Am Heart J. 2006; 152 (5):940, e941-948. [PubMed: 17070164]

Good, B.; Good, M-J. The meaning of symptoms: a cultural hermeneutic model for clinical practice. In: Eisenberg, L.; Kleinman, A., editors. The Relevance of Social Science for Medicine. D. Reidel Publishing Company; Dordrecht Holland: 1981. p. 165-196.

Heo S, Doering LV, Widener J, Moser DK. Predictors and effect of physical symptom status on health-related quality of life in patients with heart failure. Am J Crit Care. 2008; 17 (2):124-132. [PubMed: 18310649]

Jiang H, Ge J. Epidemiology and clinical management of cardiomyopathies and heart failure in China. Heart. 2009; 95 (21):1727-1731. [PubMed: 19318343]

Johnson TJ, Basu S, Pisani BA, Avery EF, Mendez JC, Calvin JE Jr, Powell LH. Depression predicts repeated heart failure hospitalizations. J Card Fail. 2012; 18 (3):246-252. [PubMed: 22385946]

Jurgens CY, Moser DK, Armola R, Carlson B, Sethares K, Riegel B. Symptom clusters of heart failure. Res Nurs Health. 2009; 32 (5):551-560. [PubMed: 19650069]

Lee KS, Song EK, Lennie TA, Frazier SK, Chung ML, Heo S, Wu JR, Rayens MK, Riegel B, Moser DK. Symptom clusters in men and women with heart failure and their impact on cardiac event-free survival. Journal of Cardiovascular Nursing. 2010; 25 (4):263-272. [PubMed: 20539161]

Miaskowski C, Aouizerat BE, Dodd M, Cooper B. Conceptual issues in symptom clusters research and their implications for quality-of-life assessment in patients with cancer. J Natl Cancer Inst Monogr. 2007; (37):39-46. [PubMed: 17951230]

Rector TS, Kubo SH, Cohn JN. Patients' self-assessment of their congestive heart failure, Part 2: Content, reliability and validity of a new measure, the Minnesota Living with Heart Failure Questionnaire. Heart failure. 1987; 3:198-209.

Rhodes VA, Watson PM. Symptom distress--the concept: past and present. Semin Oncol Nurs. 1987; 3 (4):242-247. [PubMed: 3321273]

Roger VL, Go AS, Lloyd-Jones DM, Benjamin EJ, Berry JD, Borden WB, Bravata DM, Dai S, Ford ES, Fox CS, Fullerton HJ, Gillespie C, Hailpern SM, Heit JA, Howard VJ, Kissela BM, Kittner SJ, Lackland DT, Lichtman JH, Lisabeth LD, Makuc DM, Marcus GM, Marelli A, Matchar DB, Moy CS, Mozaffarian D, Mussolino ME, Nichol G, Paynter NP, Soliman EZ, Sorlie PD, Sotoodehnia N, Turan TN, Virani SS, Wong ND, Woo D, Turner MB. Heart disease and stroke statistics--2012 update: a report from the American Heart Association. Circulation. 2012; 125 (1):e2-e220. [PubMed: 22179539]

Ryan CJ, DeVon HA, Horne R, King KB, Milner K, Moser DK, Quinn JR, Rosenfeld A, Hwang SY, Zerwic JJ. Symptom clusters in acute myocardial infarction: a secondary data analysis. Nurs Res. 2007; 56 (2):72-81. [PubMed: 17356437] 
Smith OR, Gidron Y, Kupper N, Winter JB, Denollet J. Vital exhaustion in chronic heart failure: symptom profiles and clinical outcome. J Psychosom Res. 2009; 66 (3):195-201. [PubMed: 19232231]

Smith OR, Kupper N, Schiffer AA, Denollet J. Somatic depression predicts mortality in chronic heart failure: can this be explained by covarying symptoms of fatigue? Psychosom Med. 2012; 74 (5): 459-463. [PubMed: 22511727]

Song EK, Moser DK, Rayens MK, Lennie TA. Symptom clusters predict event-free survival in patients with heart failure. J Cardiovasc Nurs. 2010; 25 (4):284-291. [PubMed: 20539163]

Stromberg A, Dickstein K. What is new and of special interest to nurses in the 2008 ESC guidelines for diagnosis and treatment of acute and chronic heart failure? Eur J Cardiovasc Nurs. 2008; 7 (4): 257-258. [PubMed: 19013409]

Teunissen SC, de Graeff A, de Haes HC, Voest EE. Prognostic significance of symptoms of hospitalised advanced cancer patients. Eur J Cancer. 2006; 42 (15):2510-2516. [PubMed: 16962316]

Tishelman C, Degner LF, Rudman A, Bertilsson K, Bond R, Broberger E, Doukkali E, Levealahti H. Symptoms in patients with lung carcinoma: distinguishing distress from intensity. Cancer. 2005; 104 (9):2013-2021. [PubMed: 16178002]

Zambroski CH, Moser DK, Bhat G, Ziegler C. Impact of symptom prevalence and symptom burden on quality of life in patients with heart failure. Eur J Cardiovasc Nurs. 2005; 4 (3):198-206. [PubMed: 15916924] 


\section{Research Highlights}

\section{What is already known about the topic}

- All heart failure patients have symptoms.

- Escalating symptoms, that are often not recognized, result in the frequent hospitalizations common in this population.

- Although symptoms occur simultaneously in most patients, researchers and clinicians focus on individual symptoms.

\section{What this paper adds}

- The findings from this study show that heart failure symptoms occur in identifiable clusters and we suggest that provision of this information to patients and healthcare providers may improve symptom recognition and appropriate action

- The data demonstrate that there are similar symptom clusters across of variety of cultures in the United States, Europe and Asia.

- The data from this study show that the clusters found were a physical capacity symptom cluster that consisted of dyspnea, difficulty in walking or climbing, fatigue/increased need to rest, and fatigue/low energy, and an emotional/ cognitive symptom cluster that consisted of worrying, feeling depressed, and cognitive problems. 


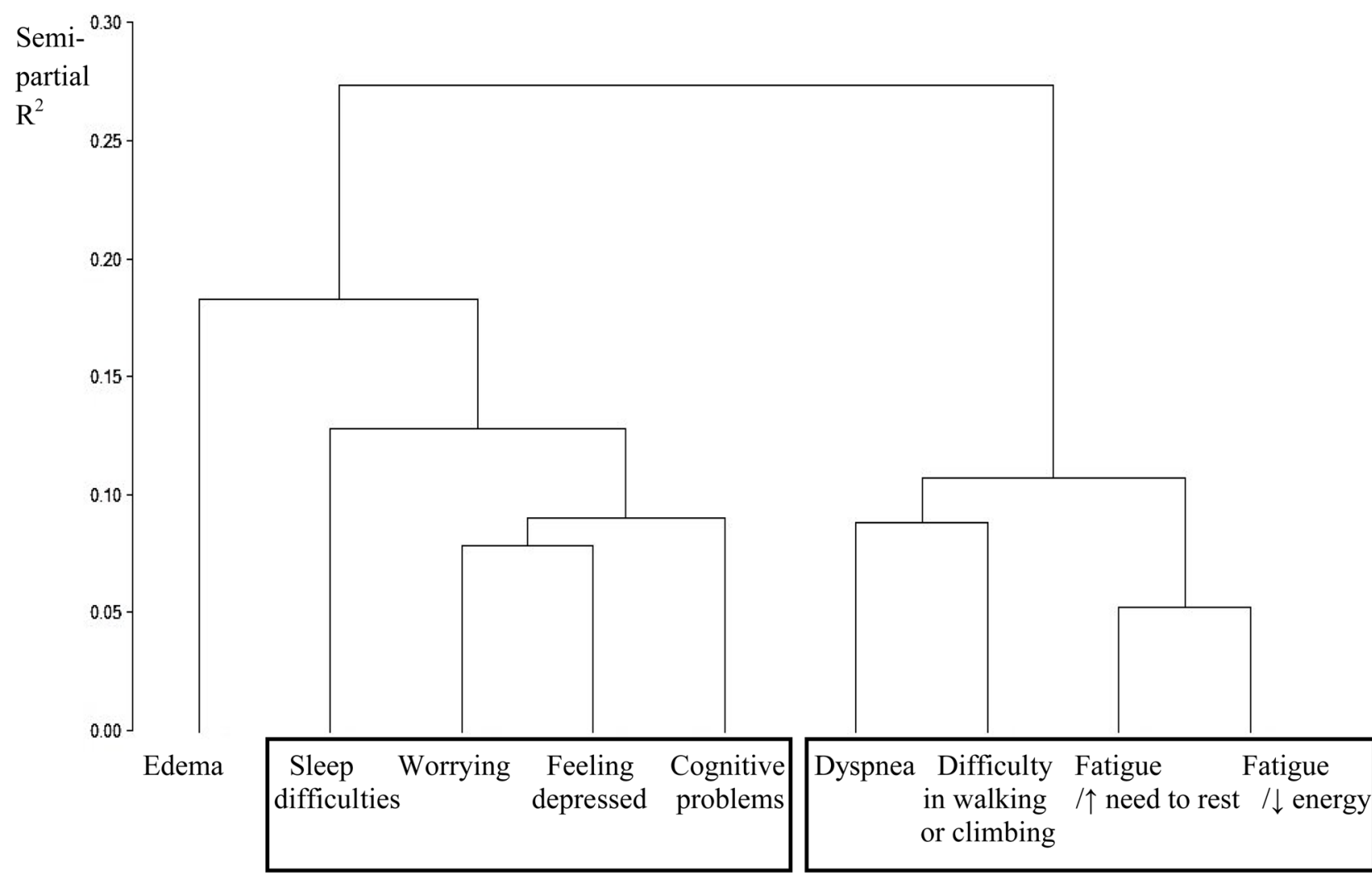

Emotional cluster

Physical cluster

Figure 1.

Dendrogram in the United States 


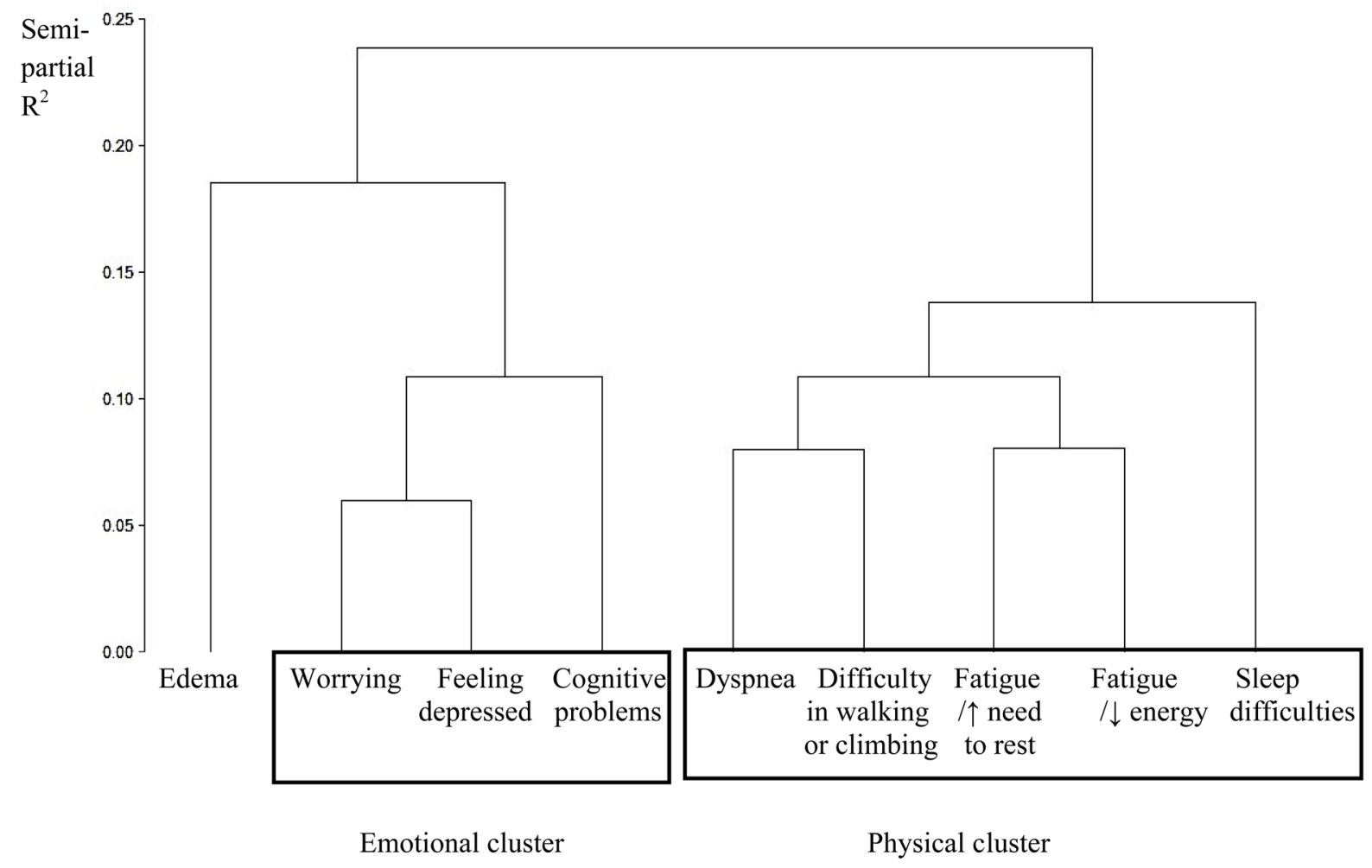

Figure 2.

Dendrogram in Asia 


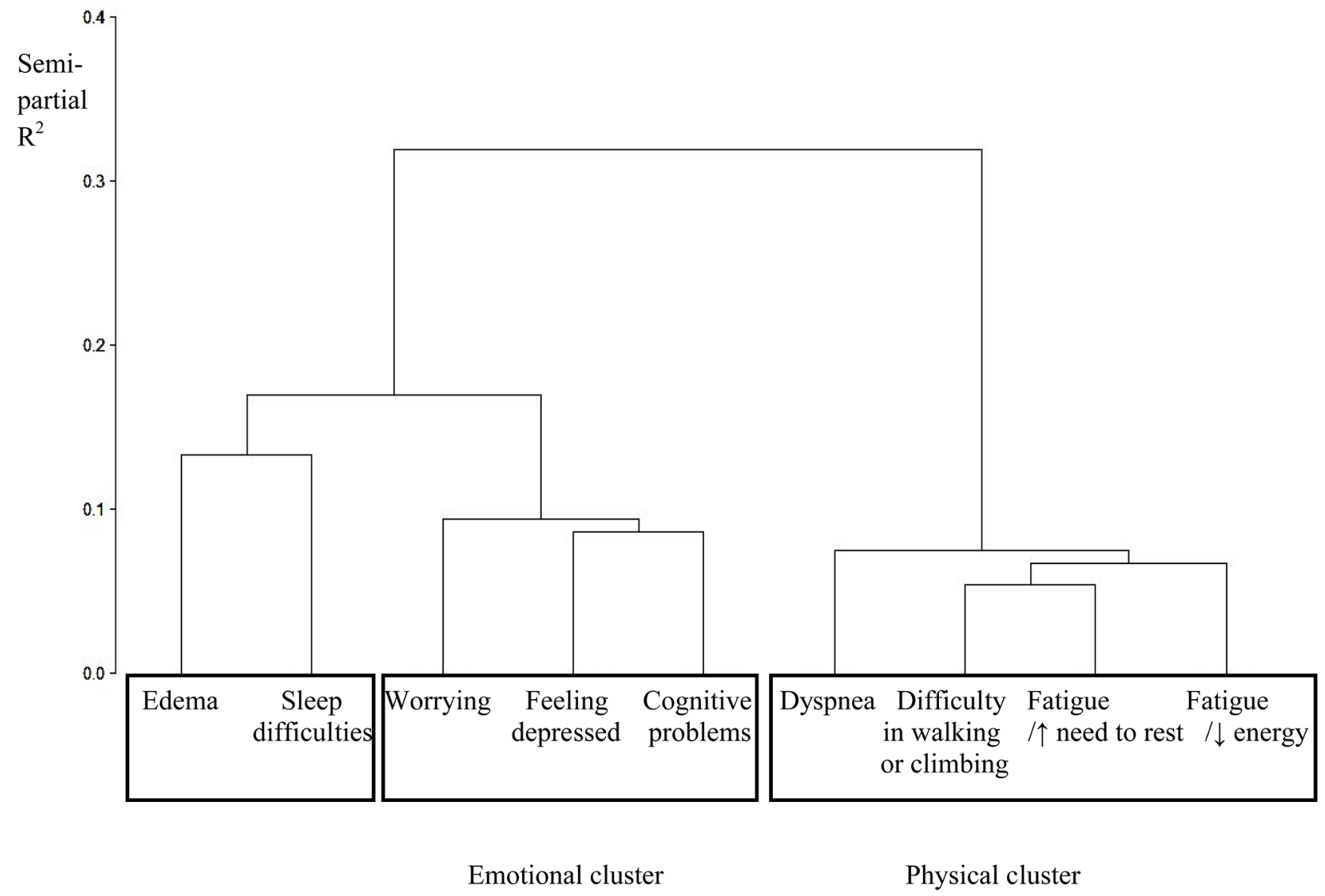

Figure 3 .

Dendrogram in Europe 
